\title{
The Premature Dropout in the Group Treatment of Bulimia
}

\author{
Cheryl A. Merrill, Ph.D. \\ Robert A. Mines, Ph.D. \\ Renee Starkey, M.A.
}

Premature dropouts in the group treatment of bulimia may be disruptive to the development of group process and cohesiveness. This article presents the results of a discriminant analysis of persisters versus dropouts across six bulimia groups. The discriminant analysis was able to correctly classify dropouts and persisters $83 \%$ of the time on demographic variables, $73 \%$ on tension and anxiety variables, and $90 \%$ of the time using cognitive variables. Specific characteristics of persisters and dropouts and treatment implications are discussed.

Bulimia is an eating disorder that appears to be increasing in prevalence among adolescent and adult women (Halmi, Falk, \& Schwartz, 1981) and that has serious medically related effects associated with it. Clinically, a group treatment approach may be particularly appropriate in working with bulimic clients owing to the secrecy and isolation that is generally associated with the condition.

As with other treatment groups, premature termination can be a problem. Clients who terminate prematurely may not have benefited from therapy. Premature termination, however, effects not only the

Cheryl A. Merrill, Ph.D. is psychotherapist, and Robert A. Mines, Ph.D. is psychologist at Mines and Associates: Counseling Psychology Services, Denver, Colorado. Renee Starkey, M.A., is a doctoral student, Counseling Psychology Program, University of Denver. An extended version of this manuscript is available with the discriminant analysis tables and a comprehensive literature review included. Requests should be sent to Dr. Robert A. Mines, Director, Mines and Associates: Counseling Psychology Services, 700 Broadway, Suite 1125. Denver, Colorado 80203. 
dropout but also other group members. The repetition necessary for new group members slows down the work of the group. Subgrouping tends to occur between original members and new members, which also decreases group cohesiveness. In addition, dropping out may affect other group members in a "wave phenomenon"; i.e., leaving seems to spread among group members, causing morale to drop within the group (Stone, Blaze, \& Bozzato, 1980). Because of these factors, the dropout phenomenon is an important consideration in the selection and retention of subjects for bulimia treatment groups. This article presents specific data on characteristics of persisters versus dropouts across a variety of bulimia treatment groups.

\section{METHODS}

\section{Subjects}

The subjects included in the original therapy groups were 53 women from 18 to 48 years of age who met the DSM-III (1980) criteria for bulimia. One subject was Oriental, the remainder were Caucasian. Of the original 53 subjects, 20 dropped out prior to the twentieth week of therapy.

\section{Assessment}

The following assessment instruments were administered to all of the original group members. The Behavioral Analysis History Questionnaire (Cautela, 1970) provided demographic information about subjects (e.g., age, sex, weight, race, previous treatment history, marital status, number of siblings, educational level, employment, sexual history, and religion). It also provided a measure of frequency of negative cognitions. Additional assessment instruments administered included the Cues for Tension and Anxiety Survey Schedule (CTASS) (Cautela, 1973a), the Thought Stopping Survey Schedule (TSSS) (Cautela and Upper, 1975), the Assertive Behavior Survey Schedule (ABSS) (Cautela, 1973b), the Beck Depression Inventory (BDI) (Beck, 1979), and the Restraint Measurement Scale (Herman, 1978).

\section{Procedure}

The original study consisted of one traditional, unstructured psychotherapy group, three cognitive-behavioral groups, and two feminist groups. Four groups had advanced doctoral students as cotherapists; one cogritive-behavioral group was led by a faculty member and a Master's level graduate student; and one cognitive-behavioral group 
was led by two Master's level students. The groups ran from 30 to 40 weeks.

One of the 20 subjects who terminated prior to the twentieth week of treatment could not be contacted because she had moved and left no forwarding address. A cover letter and dropout questionnaire were sent to the 19 subjects who could be contacted. Of these 19 subjects, $17(89 \%)$ completed and retumed the dropout questionnaire.

\section{Analysis}

Frequency analyses and discriminant analyses were performed to determine which variables on the various assessment instruments discriminated between the subjects who dropped out and those who remained in therapy. The standardized weighted coefficients were used to determine the relative importance of each of the variables in the discriminant function, with the largest coefficient (whether regative or positive) contributing the most variance to the discriminant function. A potent variable may have been assigned a low coefficient or not have been included in the function if it was highly correlated with another variable that was entered into the function first. The square of the canonical correlation was used to estimate the variance accounted for by the predictor variables.

If the discriminant function was effective, a high percentage of cases were correctly classified as having dropped out or remained. The probability of being assigned to the dropout group or the persister group on the basis of chance was compared with the classification of the cases using the discriminant function in order to determine the effectiveness of the discriminant function.

\section{RESULTS}

\section{Frequency Analysis}

Of the original 53 members, $33(62.3 \%)$ remained past the twentieth week; 20 subjects $(37.7 \%)$ dropped out prior to the twentieth week. Of the 17 dropouts who returned questionnaires, 10 reported no change in their binge and purge frequencies; 7 reported improvement; and no one reported getting worse.

Of the 6 groups, 3 had dropout rates of approximately $50 \%$, whereas the remaining 3 groups lost only 1 or 2 members (out of 6 or 7 total members). The groups with the lower dropout rate had the more experienced leaders. The dropout subjects gave a variety of reasons for early termination from the groups. The most common reason given was that the subject did not believe the group could help her bulimia. 
Other reasons given for early termination included frustration over perceiving no change; feeling that they did not fit in with the other group members; and being afraid to interact in the group. Two additional reasons that were given as contributing to their decision to drop out included financial concerns and receiving individual, family, or group treatment elsewhere.

\section{Discriminant Analysis}

\section{Demographic Data}

Several demographic factors appeared to significantly differentiate between the dropouts and persisters. The variables that appear to contribute most to the discriminant function were whether or not the subjects were working, current sexual activity, difficulty in going to sleep, and marital status.

The variable with the highest standardized discriminant function coefficient was whether or not the subject was working; persisters were more likely to be employed than were dropout subjects. Dropouts were less likely to be sexually active, less likely to be married, and tended to be younger than persisters. They were also less likely to have difficulty going to sleep at night, which may relate to less subjective discomfort.

Using demographic variables the discriminant function classified $83 \%$ of the subjects correctly. Of those remaining in treatment, $81.8 \%$ were correctly predicted, whereas $18.2 \%$ were false negatives. Of the dropouts, $85 \%$ were correctly predicted, with $15 \%$ false positives. The canonical correlation was 0.68 , accounting for approximately $46 \%$ of the variance.

\section{Cognitive and Anxiety Data}

The results of the discriminant analyses performed on the dropout data indicated that both the Cues for Tension and Anxiety Survey Schedule and the Thought Stopping Survey Schedule were useful in predicting whether subjects were dropouts or persisters.

On the CTASS, dropouts were more likely to manifest respiratory and muscular tension, whereas persisters reported more cardiovascular and gastrointestinal tension. Using CTASS scores the discriminant function was able to classify $73.58 \%$ of the subjects correctly. Of those remaining in treatment, $63.6 \%$ were correctly predicted, with only $10 \%$ false positives. The canonical correlation was 0.57 , accounting for approximately $33 \%$ of the variance.

On the TSSS, subjects remaining in treatment tended to have more negative cognitions than did dropout subjects. These cognitions fit Beck's (1979) triad of negative view of self, the world, and the future. 
The discriminant analysis correctly classified $90 \%$ of the cases. Of those remaining in treatment, $87.9 \%$ were correctly classified, whereas $12.1 \%$ were false negatives. Of the dropouts, $95 \%$ were correctly classified, with only $5 \%$ false positives. The first discriminant function for the Thought Stopping Survey Schedule has a canonical correlation of 0.82 and a squared canonical of 0.67 , accounting for $67 \%$ of the variance of dropping out versus remaining.

\section{DISCUSSION}

Based on this study it appears that certain demographic information, feelings of tension and anxiety, and self-statements of subjects may be useful in predicting which subjects will remain in treatment which will drop out of the group treatment of bulimia. Other measures, including binge/purge frequencies and the Beck Depression Inventory, did not discriminate between dropouts and persisters. This could be due to being highly correlated with another variable that was entered into the function first. In the case of the Beck Depression Inventory, failure to discriminate could be due to a lack of variance among Beck scores. Even though the level of depression was not associated in this study with premature termination, the level of depression should still be assessed and considered in the screening of bulimic clients for group treatment, since they may need a referral for antidepressant medication (Pope, Hudson, Jonas, \& Yurgelun-Todd, 1983).

Demographically, dropout subjects tended to be younger than persisters, which is consistent with Sethna and Harrington's (1971) findings. They were less likely to be employed and less likely to be married or sexually active. From this information it could be hypothesized that the dropout subjects tended to be less mature and/or may have been more socially isolated. In addition the inability to commit to the therapy process may be a reflection of their lack of commitment or direction in these other life domains. These hypotheses are consistent with findings by Baekeland and Lundwall (1975) and Frank, Gliedman, Imber, Nash, \& Stone (1957).

Based on the discriminant analysis of the CTASS, it appears that the dropouts and persisters may use different physiological systems or different perceptions of those physiological systems as cues for tension and anxiety. The dropouts tended to report more respiratory and muscular tensions, whereas the persisters reported more cardiovascular and gastrointestinal tension. This, along with a tendency to have more negative cognitions, may have resulted in increased subjective discomfort and an increased motivation to remain in treatment. This is consistent with previous studies that suggest that clients must be sufficiently motivated to undergo the continued stress of the group therapeutic 
process (Grotjahn, 1982; Koran \& Costell, 1973; Nash, Frank, Gliedman, Imber, \& Stone, 1957; Sethna \& Harrington, 1971).

The cognitive variables reported more often by the persisters included self-depracatory thoughts such as "I am growing old," "I am frigid," and "I am not worthwhile." They also manifested a lack of feeling in control, with such cognitions as "I am going to panic." The frequency of such self statements as "I would like to have a homosexual experience" may reflect a disturbance of sexual identity. Persisters appeared somewhat fearful of interpersonal relationships, reflected by such statements as "Someone I care for might die," "People will find out I am a phony," "I will hever find someone to love me," "I am afraid of being left alone in life," and "I would like to hurt someone." Other negative cognitions that reflected a negative view of the world included "The world is a mess," "The future looks hopeless," and "I don't have enough money."

In an inverse sense subjects' self-efficacy estimates may have contributed to dropping out of treatment. Overall, the dropout subjects reported fewer negative cognitions than the persister subjects. For the purpose of the present study, the twentieth week of treatment was selected to distinguish dropouts from persisters based on a general time frame for significant behavioral improvement to occur in bulimic subjects in this study. Since some of the subjects who dropped out prior to the twentieth week reported that they had improved during the treatment, they may not have considered themselves premature dropouts.

An observed, but untested, phenomenon in this study was that a clinically significant number of bulimic clients had Axis II diagnoses or features of Axis II diagnoses (Mines, 1984). It was dinically observed that a greater number of the dropouts had Axis II features. This is an important consideration in screening bulimic dients for group treatment, since secondary axis clients may drop out prematurely, be disruptive in a group treatment format, and may require a strong leadership style. Bulimic clients with Axis II diagnoses may be more appropriately treated in an individual format or an intensive day program.

An additional associational variable related to the dropout rate was the experience level of the therapist. Groups having the highest level of therapist experience generally have the lowest dropout rates. This finding is consistent with findings in a number of studies on dropouts from individual therapy (Baekeland \& Lundwall, 1975; Baum, Felzer, D'Zmura, \& Shumaker, 1966; Luborsky, Auerback, Chandler, \& Cohen, 1971; McNair, Lorr, \& Callahan, 1963; Meyers \& Auld, 1955; Sullivan, Miller, \& Smelser, 1958).

In summary, the dropouts were younger, unmarried, unemployed, and had less noticeable physical tension and more positive cognitive 
beliefs than the persisters. These variables could be accounted for by the hypothesis that the dropouts were less able to make commitments to others or that they had not experienced their disorder long enough to be in as severe distress as the persisters. In addition, the level of experience of the therapist was associated with persistence. Given the complexity and associated pathology exhibited by bulimic clients, it seems reasonable that the more experienced therapists would have a lower dropout rate because they could manage the clients more effectively. In this study the most experienced leaders had the better outcome.

Based on the data collected from group members within this study, it appears that bulimia treatment groups that are time-limited and structured may be most helpful to bulimic clients (Mines, 1984). Two frequent reasons given for premature termination of therapy were the subject's perceptions that they could not be helped and a feeling of not belonging in the group. It appears that the instillation of hope and developing a sense of universality are important initially when working with bulimia treatment groups. It is suggested that the treatment groups should focus on concrete skill changes related to changing the bulimic subjects' assumptions or beliefs and self-statements related to body image and dietary rules. In addition, the therapy groups should focus on the management of physiological symptoms related to tension and anxiety and on the behavioral management of the binge/purge phenomena until more specific group treatment studies separating out these components are reported.

\section{REFERENCES}

American Psychiatric Association (1980). Diagnostic and Statistical Manual of Mental Disorders (3rd ed.). Washington, D.C.: Author.

Baekeland, F., \& Lundwall, L. (1975). Dropping out of treatment: A critical review. Psychological Bulletin, 82, 738-783.

Baum, O., Felzer, S., D'Zmura, T., \& Shumaker, E. (1966). Psychotherapy, dropouts and lower socioeconomic patients. American Joumal of.Orthopsychiatry, 36, 629-635.

Beck, A. (1979). Beck Depression Inventory. In A. Beck, A. Rush, B. Shaw, \& G. Emery (Eds), Cognitioe Therapy of Depression. New York: Guilford Press.

Cautela, J. R. (1970). The behavioral analysis history questionnaire. Unpublished manuscript, Boston College.

Cautela, J. R. (1973a). The cues for tension and anxiety survey schedule. Unpublished survey schedule, Boston College.

Cautela, J. R. (1973b). The assertive behavior survey schedule. Unpublished survey schedule, Boston College.

Cautela, J. R. \& Upper, D. (1975). The process of individual behavior therapy. In M. Herson, R. M. Ersler, \& P. M. Miller (Eds.). Progress in Behavior Modification. Vol I. New York: Academic Press.

Frank, J. D., Gliedman, L. H., Imber, S. D., Nash, E. H., \& Stone, A. R. (1957). Why patients leave psychotherapy. Archives of Neurology and Psychiatry, 77, 283-299.

Grotiahn, M. (1982). Leaming from dropout patients: A clinical view of patients who discontinue group psychotherapy. International joumal of Group Psychotherapy, 22, 306319. 
Halmi, K. A., Falk, J. P., \& Schwart, E. (1981). Binge eating and vomiting: A survey of a college population. Psychological Medicine, 11, 697-706.

Herman, C. P. (1978). Restrained eating. Psychiatric Clinics of North America, 1, 593-607.

Koran, L. M., \& Costell, R. M. (1973). Early termination from group psychotherapy. International Joumal of Group Psychotherapy. 23(3), 346-359.

LuborskF, L., Auerback, A. H., Chandler, M., \& Cohen, J. (1971). Factors influencing the outcome of psychotherapy: A review of quantitative research. Psychological Bulletin, 75, 145-185.

McNair, D. M., Lorr, M., \& Callahan, D. M. (1963). Patient and therapist influences on quitting psychotherapy. Journal of Consulting Psychology, 27, 10-17.

Mines, R. A. (1984). The group treatment of bulimia: A cognitive-behavioral approach. Paper presented at national meeting of American Association for Counseling and Development, Houston, Texas.

Myers, J. K., \& Auld, F. (1955). Some variables related to outcome of psychotherapy. Journal of Clinical Psychology, 11, 51-54.

Nash, E. H., Frank, J. D. Gliedman, L. H., Imber, S. D., \& Stone, A. R. (1957). Some factors related to patients remaining in group psychotherapy. International journal of Group Psychotherapy, 7, 264-274.

Pope, H., Hudson, J., Jonas, J., \& Yurgelun-Todd, D. (1983). Bulimia treated with imipramine: A placebo-controlled, double-blind study. American Journal of Psychiatry, 140, $554-558$.

Sethna, E., \& Harrington, J. (1971). A study of patients who lapse from group psychotherapy. British Jourmal of Psychiatry, 119, 59-69.

Stone, W. M., Blaze, M., \& Bozzuto, J. (1980). Late dropouts from group psychotherapy. American joumal of Psychotherapy, 34, 401-413.

Sullivan, P. L., Miller, C., \& Smeiser, W. (1958). Factors in length of stay and progress in psychotherapy. Joumal of Consulting Psychotherapy, 22, 1-9. 